\title{
Ein Beitrag zur Kenntnis des Vernins.
}

\author{
Von
}

E. Schulze.

(Aus dem agriculturchemischen Laboratorium des Polytechnikums in Zürich.)

(Der Redaktion zugegangen am 1. April 1910.)

Von den in den Pflanzen von mir entdeckten Stickstoffverbindungen ist das $\mathrm{Vernin}$ diejenige, welche am schwierigsten in einer zur eingehenden Untersuchung hinreichenden Quantität gewonnen werden konnte. Zwar fanden wir diesen Stoff in zehn verschiedenen Objekten, nämlich in jungen grünen Pflanzen von Vicia sativa, Lupinus albus und Trifolium pratense, in etiolierten Keimpflanzen von Cucurbita Pepo, in reifen Samen von Lupinus luteus und Arachis hypogaea, in unreifen Samen von Pisum sativum, im Blütenstaub von Corylus avellana und Pinus silvestris, sowie im Mutterkorn. ${ }^{1}$ ) Aber nicht nur war der Verningehalt dieser Objekte stets sehr gering, sondern es zeigte sich auch, daß Materialien, die bei der ersten Verwendung Vernin lieferten, später versagten. Dies gilt z. B. für die jungen Pflanzen von Vicia sativa. Dieses Material, in welchem das Vernin entdeckt wurde, lieferte bei der ersten Untersuchung diese Stickstoffverbindung in nicht unbedeutender

1) Diese Zeitschrift, Bd. X, S. 80 und 326, sowie Bd. XLI, S. 455; Landwirtschaftliche Versuchsstationen, Bd. XXXIII, S. 89. Aus einigen der obengenannten Objekte wurde das Vernin nur in sehr kleiner Menge erhalten. Wir haben stets konstatiert, daß die von uns für Vernin erklärten Substanzen das recht charakteristische Aussehen dieser Stickstoffverbindung besaßen und in den Reaktionen mit ihr übereinstimmten; auch wurde stets nachgewiesen, daß diese Substanzen beim Erhitzen mit verdünnten Mineralsäuren Guanin lieferten. Nachdem festgestellt worden war, daß das Vernin beim Erhitzen mit Phloroglucin und Salzsäure die Pentosanreaktion gibt, haben wir mit allen von früher her noch in unserem Besitze befindlichen Verninpräparaten diese Reaktion angestellt. Die Identität aller jener Präparate wurde also soweit sichergestellt, wie die Verhältnisse es gestatteten. 
Quantität; wenigstens war die Ausbeute daran größer als aus irgend einem anderen Material. Als wir aber später aus Pflanzen der gleichen Art, die in verschiedenen Vegetationsstadien verwendet wurden, Vernin zu gewinnen suchten, erhielten wir diesen Stoff entweder gar nicht oder doch nur in äußerst geringer Quantität. Åhnlich war es bei den Samen von Lupinus luteus und beim Mutterkorn; wir erhielten Vernin nur aus je einem Muster dieser Materialien, aus den später untersuchten Mustern dagegen nicht. Es scheint also, daß diese Stickstoffverbindung sich in den Pflanzen zwar häufig bildet, später aber wieder verbraucht wird und sich infolge davon nicht anhäuft.

Am gleichmäßigsten war der Verningehalt bei $2^{1 / 2}$ bis 3 wöchentlichen etiolierten Keimpflanzen von Cucurbita Pepo. ${ }^{1}$ ) Aus diesem Material ist das Vernin dargestellt, das wir für die weiter unten beschriebenen Versuche verwendeten. Wir mußten aber eine sehr große Anzahl von Kulturen solcher Keimpflanzen verarbeiten, um 1,8 g Vernin, die für jene Versuche uns zur Verfügung stehende Quantität, zu gewinnen.

Das für die erste, von E. Bosshard und mir's) ausgeführte Untersuchung verwendete Vernin war aus jungen Pflanzen von Vicia sativa dargestellt worden. Wir wiesen damals nach, daB diese Stickstoffverbindung beim Erhitzen mit verdünnter Salzsäure Guanin liefert. Die uns zur Verfügung stehende Verninquantität war aber nicht groß genug, um feststellen zu können, was für ein Produkt neben Guanin aus dem Vernin entstand. 3) Nachdem N. Castoro und ich4) im Jahre 1904 aus den Samen von Lupinus luteus und Arachis hypogaea eine kleine Quantität von Vernin dargestellt hatten, suchten wir

1) Die Ausbeute war auch hier schwankend, doch lieferten alle Kulturen solcher Keimpflanzen Vernin.

2) Diese Zeitschrift, Bd. X, S. 80.

3) Die bei Zersetzung des Vernins erhaltene Flüssigkeit wurde damals auf Zucker geprüft, doch mit negativem Erfolge. Der Grund für dieses negative Resultat liegt wahrscheinlich darin, daß durch die ziemlich starke Salzsäure, die wir in den ersten Versuchen auf das Vernin einwirken ließen, die Pentose zersetzt worden war.

4) Diese Zeitschrift, Bd. XLI, S. 455.

Hoppe-Seyler's Zeitschrift f. physiol. Chemie. LXVI. 
diese Lücke auszufüllen. Wir fanden, daß bei der Spaltung des Vernins durch verdünnte Schwefelsäure eine Zuckerart entsteht. Daß dies eine Pentose sei, konnten wir für sehr wahrscheinlich erklären; denn das Vernin gibt beim Erhitzen mit Phloroglucin und verdünnter Salzsäure eine rein kirschrote Flüssigkeit. Wir konnten aber die Pentose nicht näher untersuchen, weil die damals zur Verfügung stehende Verninquantität zu gering war. Die bezüglichen Versuche wurden wieder aufgenommen, nachdem ich aus Keimpflanzen von Curcurbita Pepo $1,8 \mathrm{~g}$ Vernin gewonnen hatte. Zunächst wurde festgestellt, daß beim Erhitzen des Vernins mit verdünnter Salzsäure Furfur ol entsteht. Das dabei erhaltene Destillat besaß den Geruch des Furfurols und rötete ein mit Anilinacetat befeuchtetes Papier; auch gab es mit Phloroglucin eine starke Fällung. Letztere war unlöslich in Alkohol, woraus hervorgeht, daß kein Methylfurfurol entstanden war. Das Gewicht des in einem Versuche mit ca. $0,1 \mathrm{~g}$ Vernin erhaltenen Phloroglucids entsprach der Annahme, daß das Vernin ungefähr $50 \%$ Pentosan enthält. Diese Zahl kann aus verschiedenen Gründen nicht als genau bezeichnet werden; sie führt aber zu der Schlußfolgerung, daß ungefähr die Hälfte der Produkte der Hydrolyse des Vernins aus Pentose besteht. Dies steht in Übereinstimmung mit der schon im Jahre 1904 von uns (loc. cit.) ausgesprochenen Annahme, daß das Vernin ein nach der Formel $\mathrm{C}_{10} \mathrm{H}_{13} \mathrm{~N}_{5} \mathrm{O}_{5}$ zusammengesetztes Guanin-Pentosid ist.

Zur Darstellung der Pentose verwendeten wir ein Quantum von $1,223 \mathrm{~g}$ Vernin. Wir erhitzten dasselbe eine Stunde

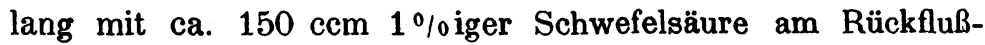
kühler. Aus der erkalteten Flüssigkeit schied sich Guaninsulfat in dünnen, zu Gruppen vereinigten Prismen aus. Entsprechend den über sein Verhalten in den Handbüchern sich findenden Angaben verlor dieses Salz bei Behandlung mit heißem Wasser die Schwefelsäure und verwandelte sich in Guanin. Die vom Guaninsulfat abgegossene Lösung wurde mit Baryumhydroxyd neutralisiert, der dabei entstandene Niederschlag, welcher noch Guanin einschloß, abfiltriert. Da das Filtrat noch etwas Guanin enthielt, so versetzten wir es mit reinem Silbersulfat. Das Fil- 
trat von dem durch dieses Reagens hervorgebrachten schwachen Niederschlage wurde durch Einleiten von Schwefelwasserstoff vom Silber befreit, dann schwach erwärmt und nun mit Baryumhydroxyd genau neutralisiert. Das Filtrat vom Baryumsulfat wurde in gelinder Wärme stark eingeengt. Es war anfangs ganz farblos, färbte sich aber schließlich etwas gelb und wurde daher mit einer kleinen Menge von Tierkohle versetzt. Die von der Kohle abfiltrierte, farblose Lösung wurde auf ein Volumen von $10 \mathrm{ccm}$ gebracht und sodann in einem Soleil-Ventzkeschen Polarisationsapparate untersucht. Sie erwies sich als linksdrehend, und zwar wurde eine Drehung von $5,5^{\circ}$ (bei 18 bis $19^{\circ}$ G.) beobachtet. Die Lösung wurde nun in einem flachen Porzellanschälchen eingedunstet, der Verdampfungsrückstand im Vacuum-Exsikkator so vollständig wie möglich ausgetrocknet. Sein Gewicht betrug 0,533 $\mathrm{g}$ (nach der Berechnung konnte die für den Versuch verwendete Verninquantität $0,579 \mathrm{~g}$ Pentose liefern; die Differenz erklärt sich aus unvermeidlichen kleinen Verlusten). Unter der, ohne Zweifel nicht ganz genau zutreffenden Voraussetzung, daß jener Verdampfungsrückstand nach dem Trocknen kein Wasser mehr enthielt, berechnet sich für den darin enthaltenen Zucker ein spezifisches Drehungsvermögen von $-17,7^{\circ}$ (bei $18-19^{\circ}$ C.). Krystallisiert haben wir den Zucker bis jetzt nicht erhalten können. Selbstverständlich kann die vorstehende Zahl nicht als ein genauer Ausdruck für das spezifischeDrehungsvermögen des im Sirup vorhandenen Zuckers hingestellt werden; denn dieser Sirup kann auch nach dem Austrocknen im Vacuumexsikkator kaum ganz wasserfrei gewesen sein. Wahrscheinlich liegt daher das Drehungsvermögen jenes Zuckers etwas höher.

Wir bestimmten ferner die Löslichkeit des Vernins in Wasser. Für die bezüglichen Versuche verwendeten wir zwei durch mehrfaches Imkrystallisieren gereinigte Verninproben. Sie wurden in heißem Wasser gelöst; abgewogene Quantitäten der nach 24 Stunden bei einer Temperatur von $17-18^{\circ} \mathrm{C}$. von den ausgeschiedenen Krystallen abfiltrierten Lösungen wurden in Wägegläschen eingedunstet, die Verdampfungsrückstände nach dem Trocknen gewogen. Wir erhielten folgende Zahlen: 
a) $13,0927 \mathrm{~g}$ Lösung gaben $0,0101 \mathrm{~g}$ Rückstand.

b) 13,0414 * , 0,0097 , *

Aus diesen Zahlen ergibt sich, daß ein Teil Vernin 1295 bis $1343 \mathrm{~T}$., im Mittel $1320 \mathrm{~T}$. Wasser von $17-18^{\circ}$ zur Lösung bedurfte. Das Vernin ist also in kaltem Wasser wenig löslich; in kochendem Wasser löst es sich dagegen in reichlicher Menge. Die große Differenz zwischen der Löslichkeit in kaltem und in heißem Wasser hat zur Folge, daß aus einer in der Hitze bereiteten wässerigen Verninlösung schon während des Erkaltens Krystalle in reichlicherMenge sich abscheiden.

Bei Untersuchung einer 5\% verdünnter Schwefelsäure im Soleil-Ventzkeschen Polarisationsapparat konnten wir eine Drehung nicht konstatieren; falls die Lösung optische Aktivität besaß, so war letztere doch ohne Zweifel sehr gering. Dagegen erwies sich eine Lösung des Vernins in 1/10-Normalnatronlauge als stark linksdrehend; für eine ca. $2 \%$ ige Lösung wurde $[\alpha]^{\mathrm{D}}$ bei $20^{\circ} \mathrm{C}$. $=-60^{\circ}$ gefunden.

Die im vorigen beschriebenen Versuche wurden unter Mitwirkung von Dr. G. Trier von mir ausgeführt.

Die weiter unten näher besprochene Tatsache, daß außer dem Vernin noch andere Pentoside des Guanins und verwandter Basen in den Organismen sich finden, veranlaßt mich, die bei der Identifizierung des Vernins in Betracht kommenden Eigenschaften dieses Körpers hier kurz anzugeben. Aus einer Lösung in heißem Wasser krystallisiert das Vernin schon während des Erkaltens in dünnen Nadeln oder flachen Prismen; bringt man dieselben auf ein Filter, preßt letzteres zwischen Papier und trocknet es, so bilden die Krystalle eine zusammenhängende atlasglänzende Masse, die sich wie ein Blatt vom Filter ablöst. Sie sind nach der Formel $\mathrm{C}_{10} \mathrm{H}_{13} \mathrm{~N}_{5} \mathrm{O}_{5}+2 \mathrm{H}_{2} \mathrm{O}$ zusammengesetzt. In kaltem Wasser sind sie wenig löslich (conf. die oben gemachten Angaben). Sie lösen sich sowohl in Alkalien, wie in verdünnten Mineralsäuren. Doch bedarf es zur Lösung einer ziemlich großen Quantität stark verdünnter Schwefelsäure; $20 \mathrm{~T}$. $1 \%$ iger Schwefelsäure genügen nicht zur Lösung von $1 \mathrm{~T}$. Vernin. In absolutem Alkohol lösen sie sich nicht. Die wässe- 
rige Lösung gibt mit Phosphorwolframsäure unter Zusatz von Salzsäure oder Schwefelsäure eine Fällung. Silbernitrat bringt darin einen durchsichtigen, gallertartigen Niederschlag hervor, der in Ammoniakflüssigkeit sich löst; auch Mercurinitrat gibt eine Fällung. Versetzt man eine wässerige Verninlösung mit Pikrinsäure, so scheidet sich nach kurzer Zeit ein Pikrat in kleinen Krystallaggregaten aus; unter dem Mikroskop erscheinen die Krystalle als feine, zu Büscheln oder Sternen vereinigte Nadeln (ihr Schmelzpunkt wurde bei ca. $190^{\circ}$ gefunden). Über das spezifische Drehungsvermögen des Vernins habe ich schon oben Angaben gemacht. Beim Erhitzen mit Phloroglucin und Salzsäure gibt das Vernin eine kirschrote Flüssigkeit. Durch Mineralsäuren wird es leicht hydrolysiert; schon einstündiges Erhitzen mit 1\%iger Schwefelsäure genügt, um es zu spalten. Das dabei entstehende Guanin läßt sich leicht isolieren und an seinen Reaktionen erkennen.

In den letzten Jahren sind mehrere Verbindungen von Alloxurbasen mit Pentosen beschrieben worden; es ist von Interesse, sie mit dem Vernin zu vergleichen. Ich nenne hier zunächst das von K. Andrlik ${ }^{1}$ ) aus Melasse und Melasseabfalllauge durch Erhitzen mit Kupfersulfat und Natron zur Abscheidung gebrachte Guaninpentosid. Bei der Hydrolyse liefert es neben Guanin eine linksdrehende Pentose, für welche $[\alpha]^{\mathrm{D}}=-16,7^{\circ}$ gefunden wurde. Andrlik erklärt diesen Körper für verschieden vom Vernin; in der Tat differieren die beiden Stoffe nach den vorliegenden Angaben in ihren Eigenschaften in bezug auf mehrere Punkte so bedeutend, dab sie nicht identisch sein können. Es ist nun darauf aufmerksam zu machen, daß O. E. v. Lippmann ${ }^{2}$ ) aus Melasse einen Stoff isoliert hat, den er für identisch mit Vernin erklären konnte. Es scheint also, daß Zuckerrüben verschiedener Provenienz zwei von einander verschiedene Guaninpentoside enthalten.

1) Chemiker-Zeitung, 1909, S. 637; Referat nach einem auf dem 3. internationalen Kongreß für angewandte Chemie in London im Jahre 1909 gehaltenen Vortrage.

2) Ber. d. Deutsch. chem. Ges., Bd. XXIX, S. 2653. 
Sehr ähnlich dem Vernin ist das von Levene und Jacobs ${ }^{1}$ ) durch Spaltung von Nucleinsäure dargestellte Guanosin, $\mathrm{C}_{10} \mathrm{H}_{13} \mathrm{~N}_{5} \mathrm{O}_{5}+2 \mathrm{H}_{2} \mathrm{O}$. Bei der Hydrolyse liefert dieser Körper Guanin und eine Pentose, für welche $[\alpha]^{D}=-19^{\circ}$ gefunden wurde. Das Guanosin ist in alkalischer Lösung stark linksdrehend, und zwar wurde $[\alpha]^{\mathrm{D}}=-60^{\circ}$ gefunden. Es sind uns zurzeit keine Tatsachen bekannt, die im Widerspruch mit der Annahme stehen, daß Guanosin und Vernin identisch sind; wir werden uns aber bemühen, diese Frage durch neue Versuche mit völliger Sicherheit zu entscheiden.

Ferner will ich hier noch erwähnen, daß das im Carnin enthaltene Inosin als ein Pentosid des Hypoxanthins erkannt worden ist. ${ }^{2}$ )

Zum Schluß will ich noch einige Bemerkungen über das zur Darstellung des Vernins von uns angewendete Verfahren machen. Wässerige Extrakte aus den auf Vernin zu untersuchenden Objekten wurden, nachdem sie zuvor von den durch Bleiessig fällbaren Substanzen befreit worden waren, mit einer Mercurinitratlösung versetzt. Die durch dieses Reagens hervorgebrachten Niederschläge wurden abfiltriert, mitkaltem Wasser gewaschen, zwischen Fließpapier abgepreßt, dann mittels Schwefelwasserstoff zersetzt. Die Filtrate vom Schwefelquecksilber engten wir, nachdem sie neutralisiert worden waren, auf ein geringes Volumen ein; wir trugen Sorge dafür, daß während des Eindunstens die Reaktion der Lösungen neutral blieb. Aus den stark eingeengten Flüssigkeiten schied sich nach dem Erkalten das Vernin aus, und zwar in vielen Fällen anfangs als Gallerte; letztere lieferte dann nach dem Wiederauflösen in Wasser Krystalle. Der Mercurinitratniederschlag kann neben Vernin noch manche andere Substanzen enthalten, z. B. Asparagin, Glutamin, Arginin und Tyrosin. Wegen seiner Schwerlöslichkeit in Wasser läßt sich das Vernin leicht vom Asparagin, Glutamin und Arginin trennen. Aber auch seine Trennung von dem Tyrosin, das sich speziell in den bei Verarbeitung von Kürbiskeimpflanzen

1) Ber. d. Deutsch. chem. Ges., Bd. XLII, S. 2102 und 3247 (1909).

$\left.{ }^{2}\right)$ Monatshefte für Chemie, Bd. XXIX, S. 157 (1908); Bd. XXX, S. 147 und 377 (1909). 
erhaltenen Mercurinitratniederschlägen in kleiner Menge vorfindet, bietet keine Schwierigkeit dar. Denn das Vernin scheidet sich aus den bei Zerlegung jener Niederschläge erhaltenen Lösungen meistens vor dem Tyrosin aus. Gesetzt aber, daß anfangs das Vernin durch etwas Tyrosin verunreingt ist, so kann man es davon befreien, indem man es in heißem Wasser löst und die beim Erkalten sich ausscheidenden Krystalle nach kurzer Zeit abfiltriert und zwischen Filtrierpapier abpreßt; das Tyrosin geht dann in die Mutterlauge über. Das in solcher Weise aus Kürbiskeimpflanzen dargestellte Vernin erwies sich nach mehrmaligem Umkrystallisieren aus Wasser als ganz frei von Tyrosin, wie aus seinem Verhalten gegen das Millon sche Reagens $\mathrm{zu}$ erkennen war.

Nach einem, mit einer sehr kleinen Verninmenge von mir ausgeführten Versuche kann man diese Stickstoffverbindung auch durch Erhitzen mit Kupfersulfat und Natronlauge zur Abscheidung bringen. In dem Filtrat von dem durch diese Reagenzien erhaltenen Niederschlage konnte ich Vernin nicht mehr nachweisen. Als ich den Niederschlag mittels Schwefelwasserstoff zersetzte, die durch Filtration vom Schwefelkupfer getrennte Flüssigkeit sodann mit Schwefelsäure erhitzte und hierauf mit Fehling scher Lösung prüfte, konnte die Anwesenheit eines diese Lösung reduzierenden Zuckers konstatiert werden, woraus man wohl schließen darf, daß das Vernin in den Niederschlag eingegangen war. Dasselbe scheint also in dieser Beziehung mit dem von Andrlik (loc. cit.) dargestellten Guaninpentosid übereinzustimmen.

Nachschrift. Es sei hier erwähnt, daß die in F. Czapeks Biochemie der Pflanzen an drei Stellen sich findenden Angaben über das Vernin meinen und meiner Mitarbeiter Anteil an den bezüglichen Untersuchungen nicht ganz im richtigen Lichte erscheinen lassen. Es heißt dort in Bd. II, auf S. 70: «Erwähnenswert ist seine (des Guanins) Entstehung aus Vernin bei Behandlung mit Salzsäure (Lippmann).» Daß bei der Spaltung des Vernins Guanin entsteht, ist aber nicht von 0. E. v. Lippmann, sondern von mir entdeckt worden (eine Angabe darüber ist außer in der von mir in Verbindung mit E. Bosshard 
publizierten Abhandlung früher schon in dieser Zeitschrift, Bd. IX, S. 444 gemacht worden). Zweitens heißt es in dem oben genannten Werke auf S. 80 "Vernin ist im Mutterkorn aufgefunden worden (vgl. Flückig er, l. c., S. 299).» Auch das Vorkommen von Vernin im Mutterkorn ist von uns (Diese Zeitschrift, Bd. X, S. 80) nachgewiesen worden. Drittens heißt es ebenda auf S. 179: "Eine beim Kochen mit Salzsäure Guanin liefernde Verbindung ist das Vernin, dessen Konstitution noch unbekannt ist und welches in geringer Menge von Schulze und Bosshard recht verbreitet in Keimlingen aufgefunden worden ist»... . ; (dann folgt eine Beschreibung der Eigenschaften des Vernins). Wer diese Angaben nacheinander liest, wird daraus nicht mit Sicherheit erkennen können, von wem das Vernin entdeckt und zuerst auf seine Spaltungsprodukte untersucht wurde. Es wird aber leicht sein, in einer neuen Auflage des oben genannten, geschätzten Werkes diese Angaben so zu ergänzen, daß in bezug auf jenen Punkt keine Unsicherheit bleibt. $\mathrm{Zu}$ der dritten Angabe bemerke ich noch, daß es nicht Keimlinge, sondern junge grüne Pflanzen von Vicia sativa und Trifolium pratense waren, aus denen E. Bosshard und ich Vernin darstellten; über Herkunft und Beschaffenheit dieser Pflanzen sind in den Landwirtschaftlichen Versuchsstationen, Bd. XXXIII, S. $97 \mathrm{ff}$. nähere Angaben gemacht worden. Außerdem fand ich (Journal für praktische Chemie [2] Bd. XXXII, S. 447) Vernin in etiolierten Keimpflanzen von Cucurbita Pepo. Zur Vervollständigung der auf das Vernin sich beziehenden Literaturangaben sei hier noch erwähnt, daß F. Ullik (Chemisches Zentrallblatt 1887, S. 828) im Gerstenmalz eine Substanz fand, die er für identisch mit Vernin erklären konnte. 